\title{
SBGN-ED - working with the Systems Biology Graphical Notation
}

\section{Tobias Czauderna \\ Falk Schreiber}

Leibniz Institute of Plant Genetics and Crop Plant Research (IPK)

Gatersleben
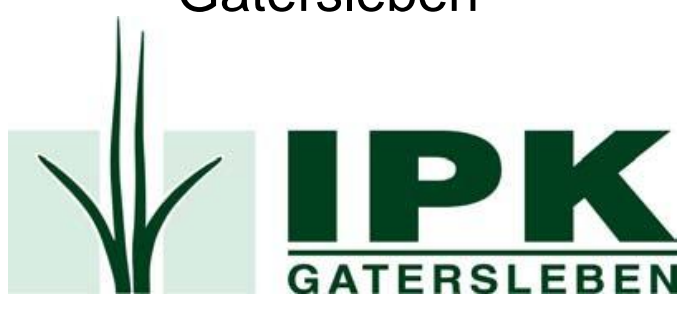

Institute of Computer Science Martin Luther University Halle-Wittenberg Halle

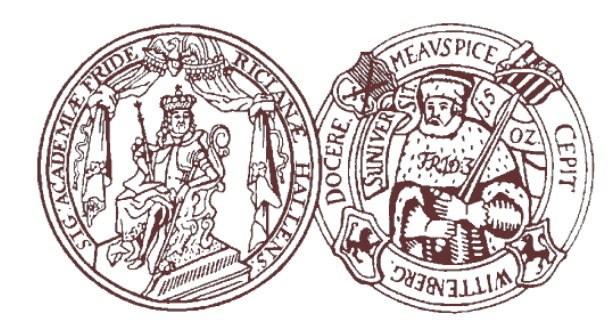




\section{Working with SBGN}

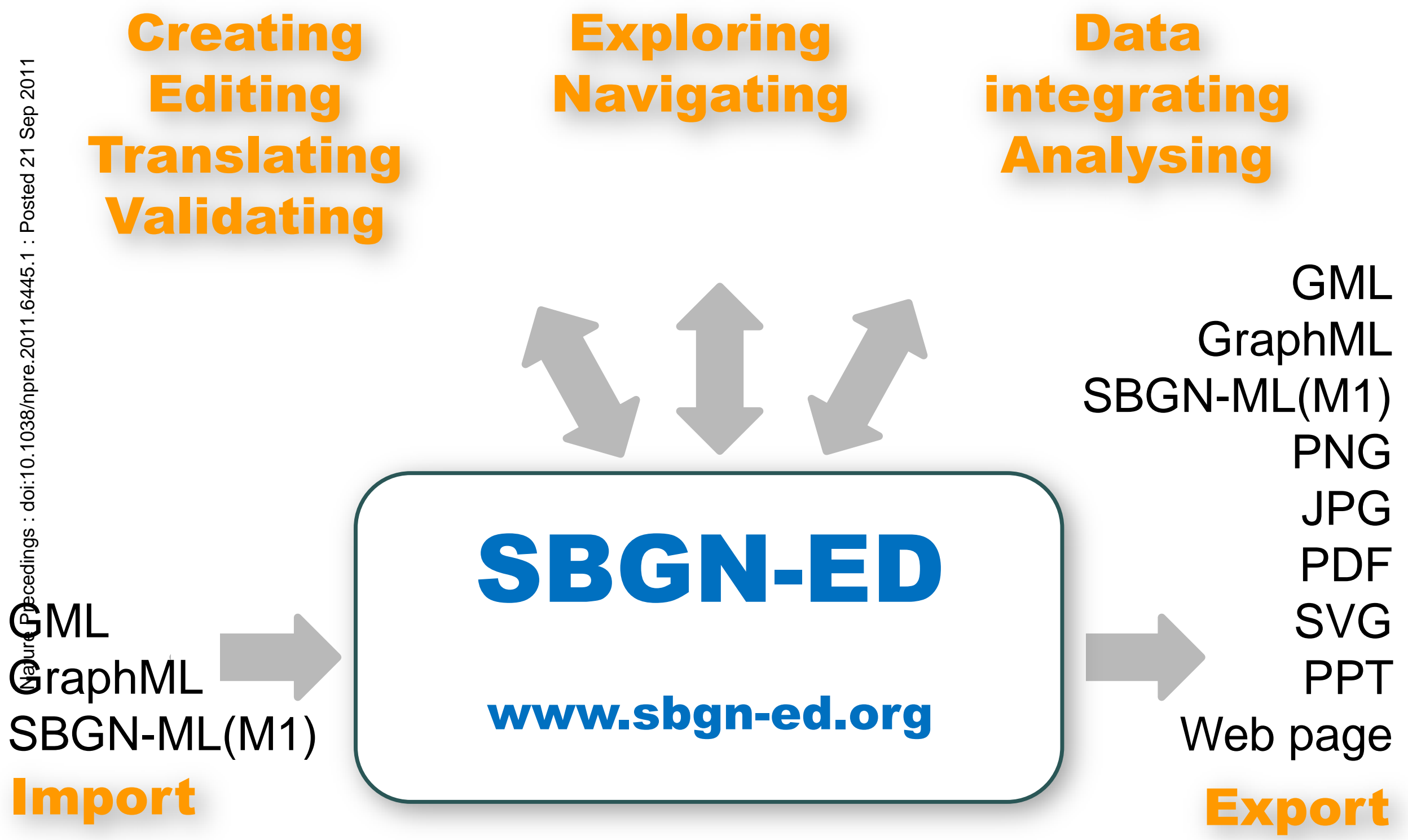




\section{Creating and Editing SBGN Maps}

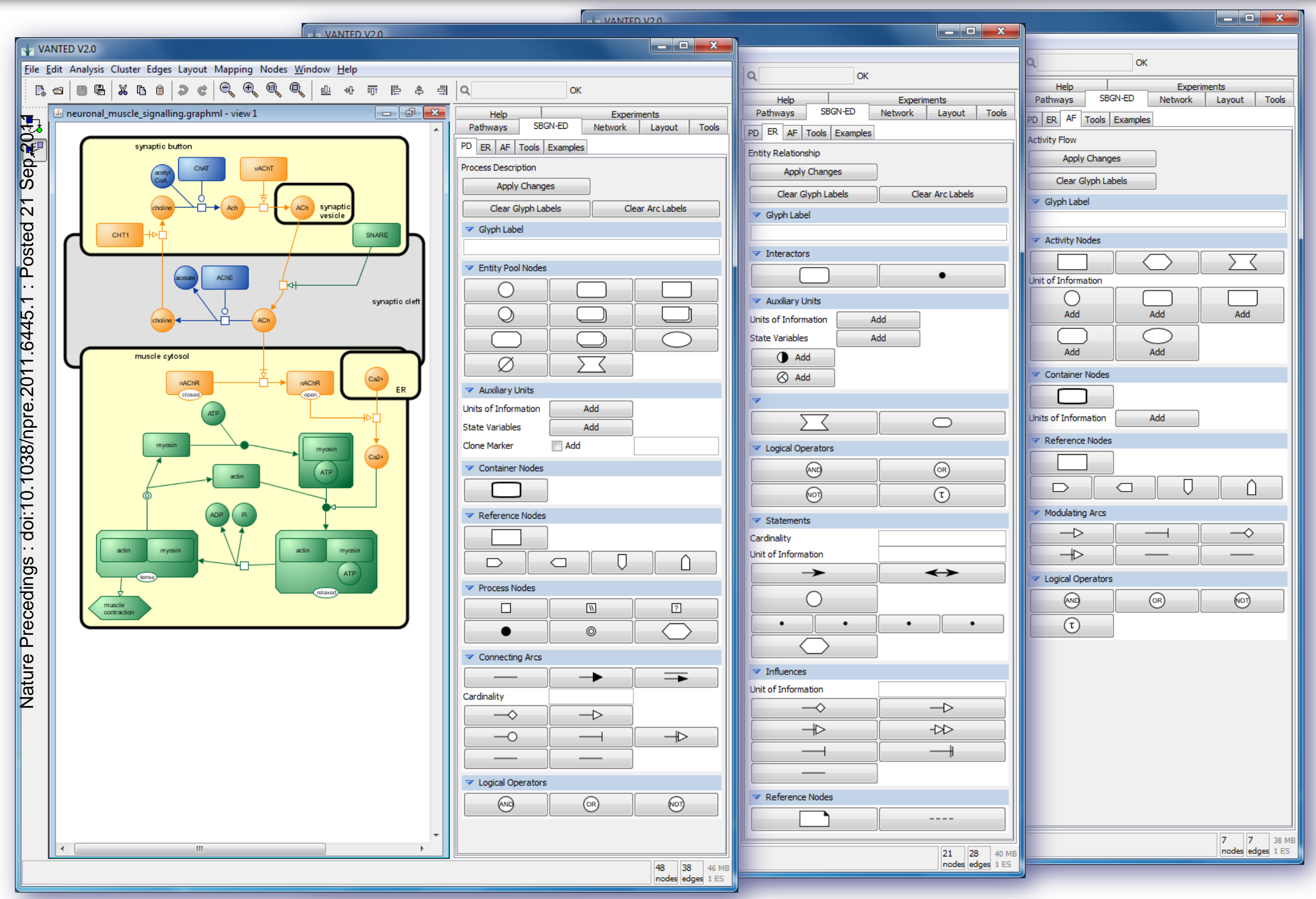




\section{Translating SBGN Maps from KEGG}

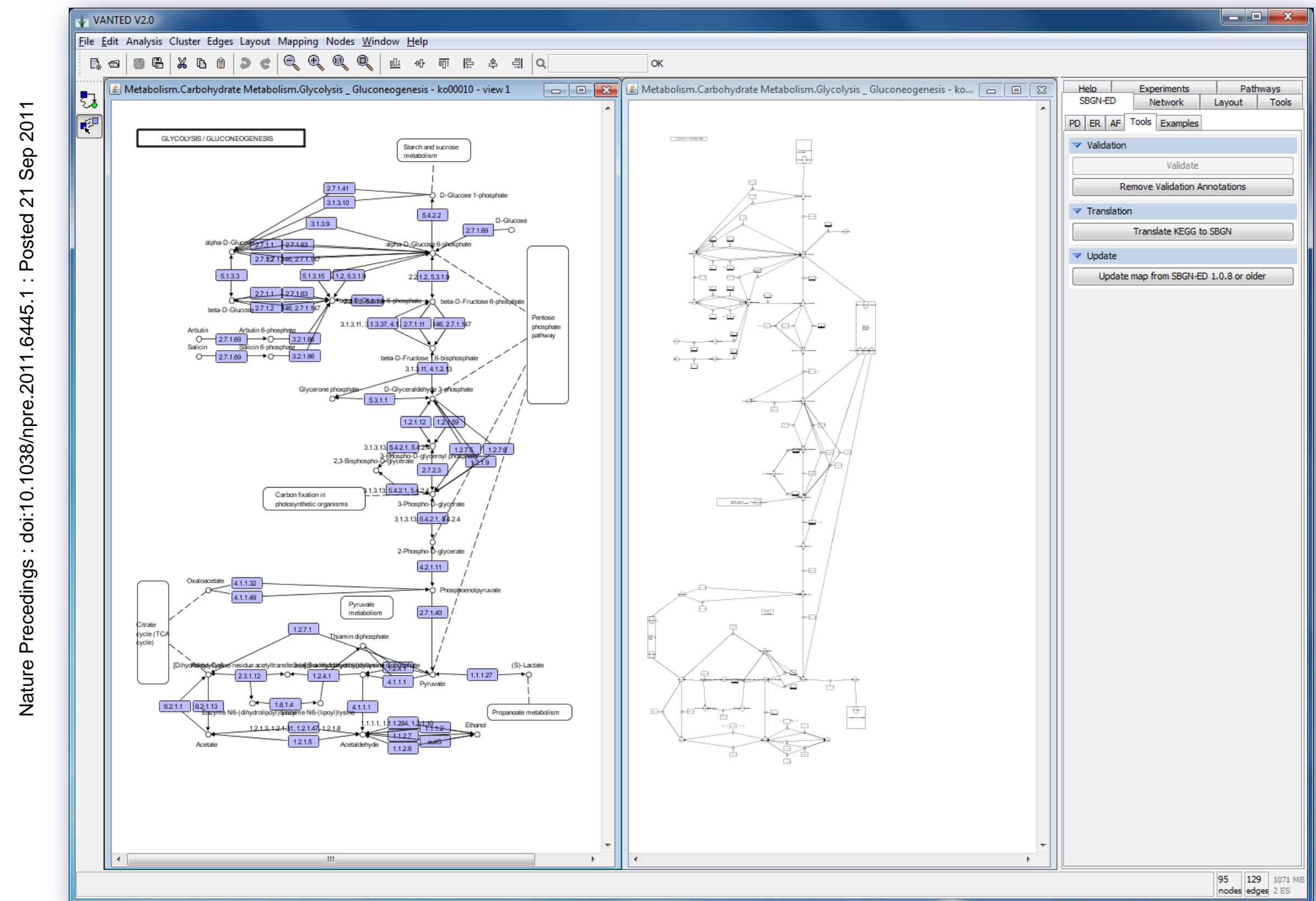




\section{Data Integrating and Analysing in SBGN Maps}

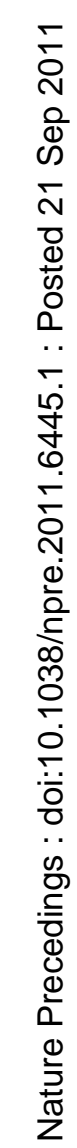
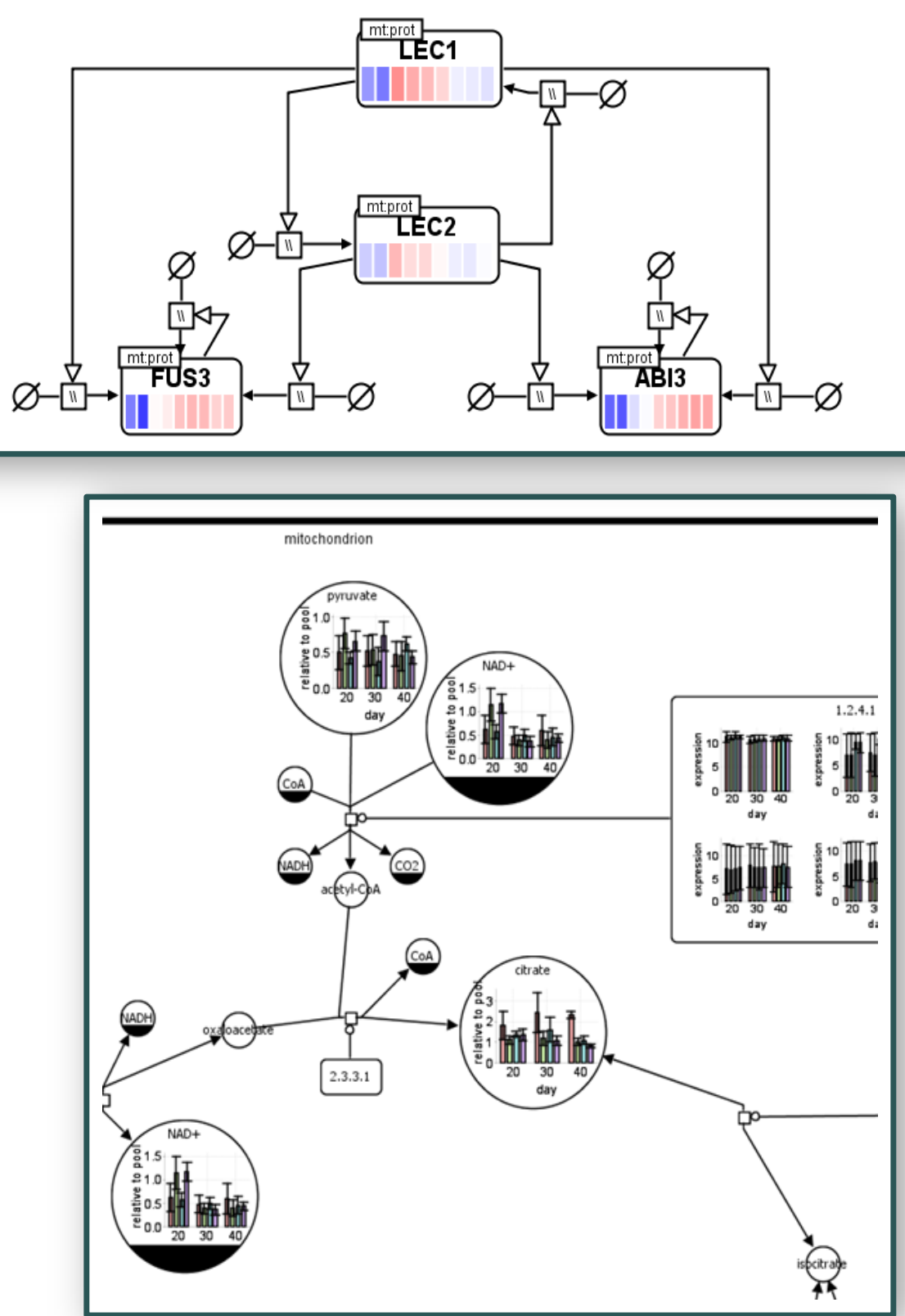
Current version (see www.sbgn-ed.org)

- Improved user interaction

- More export possibilities

- Powerpoint

- Webpages

- SBGN-ML(M1)

Next version (under development)

- More importers

- SBML (all up to L3V1) (JSBML)

- BioPAX (L2,L3) (Paxtools V4)

- Automatic layout

- More interaction possibilities

More export possibilities (SBML and BioPAX) planed, but needs validation of user changes! 


\section{Layout}

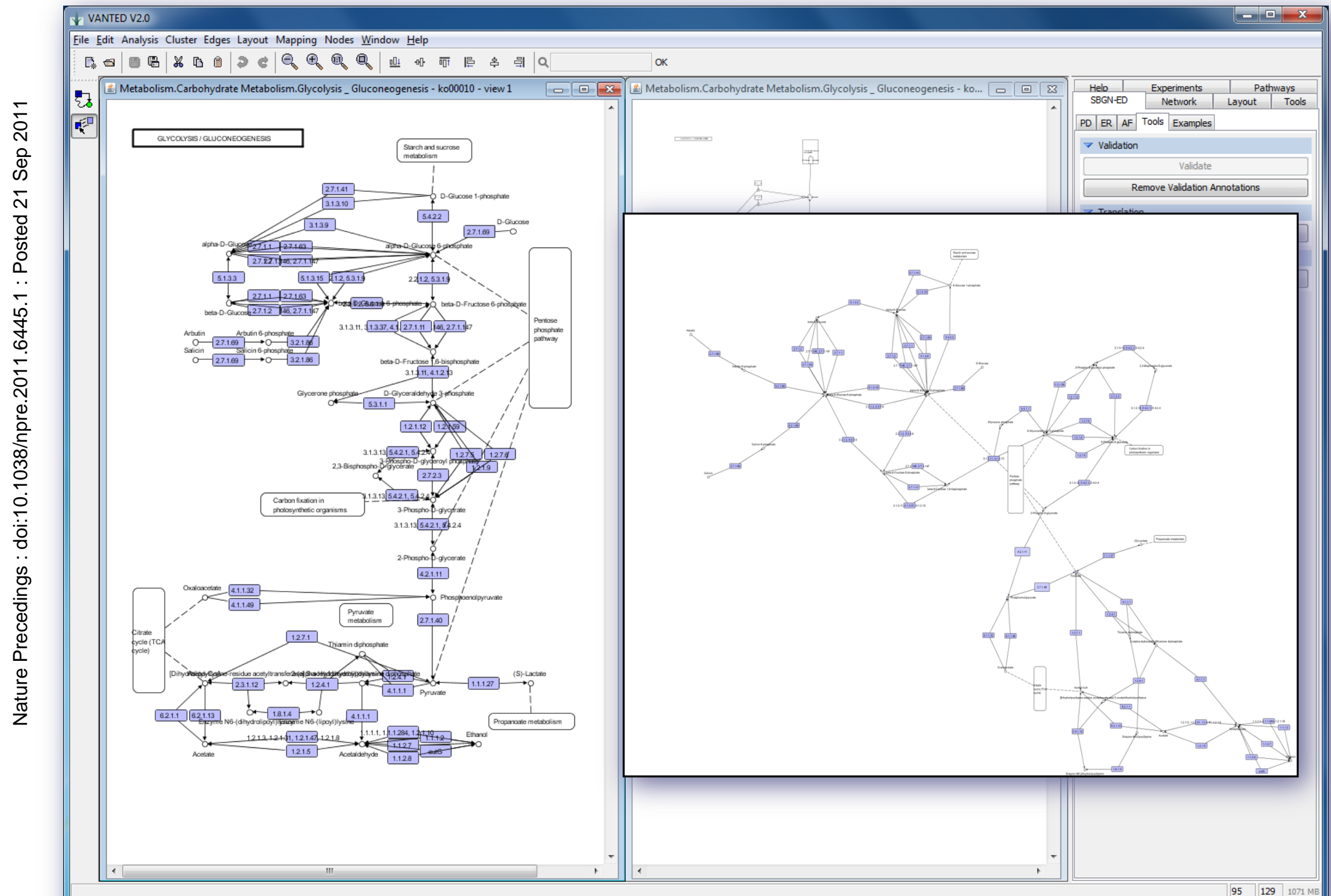




\section{Layout}

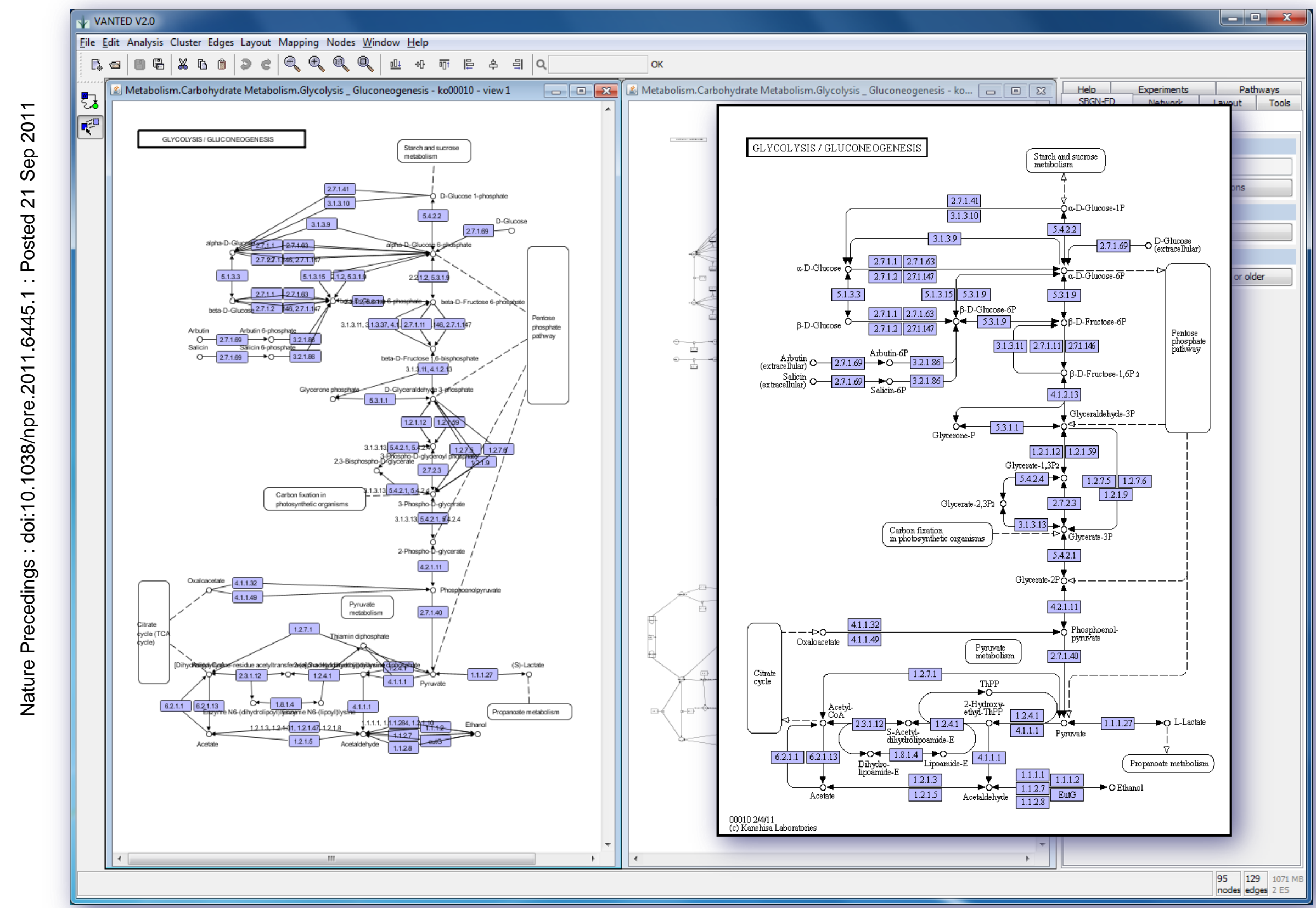




\section{Constrained KEGG Layout}
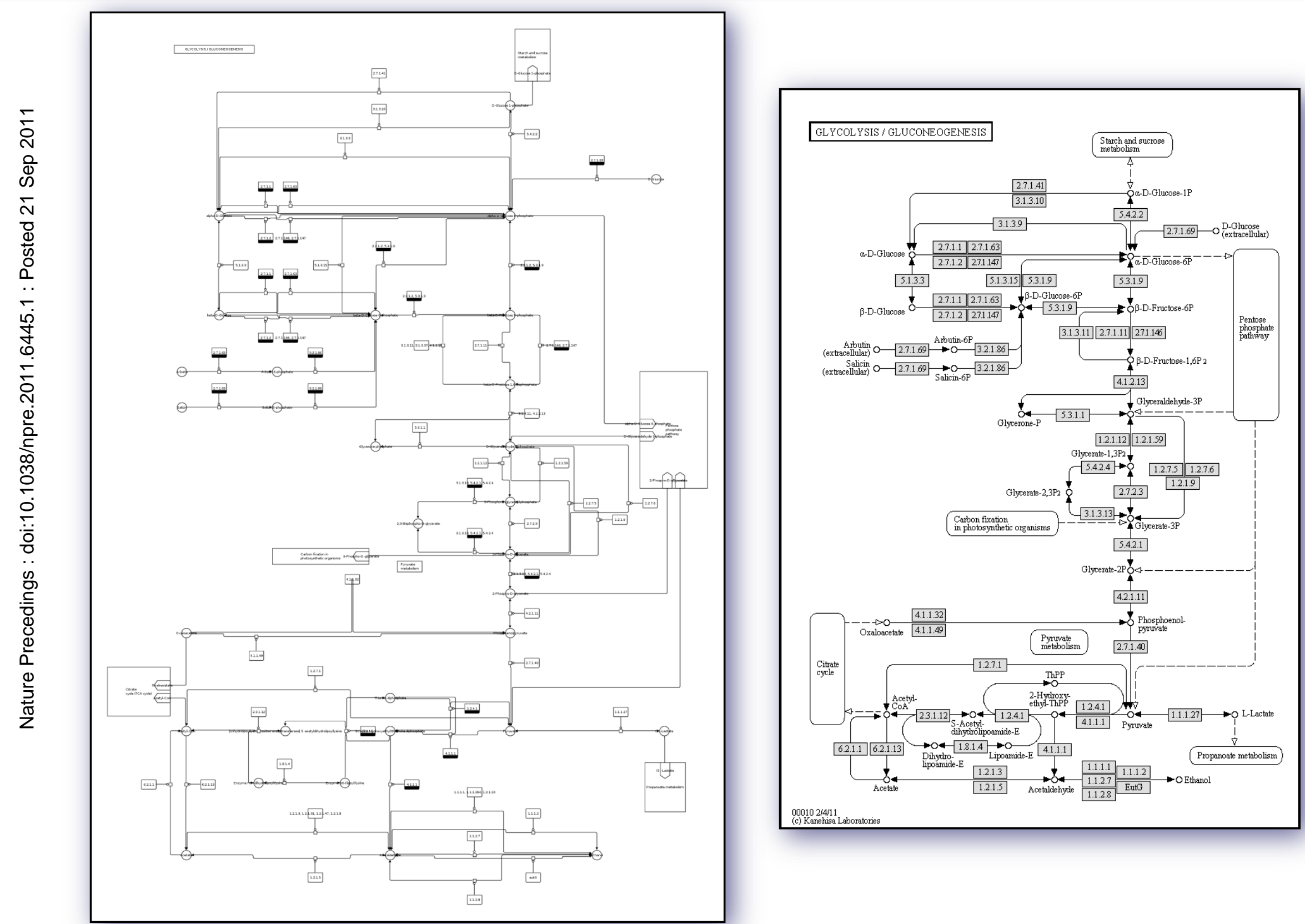


\section{Constrained KEGG Layout}

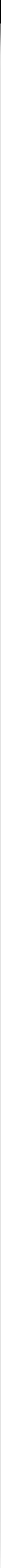




\section{Constrained KEGG Layout}

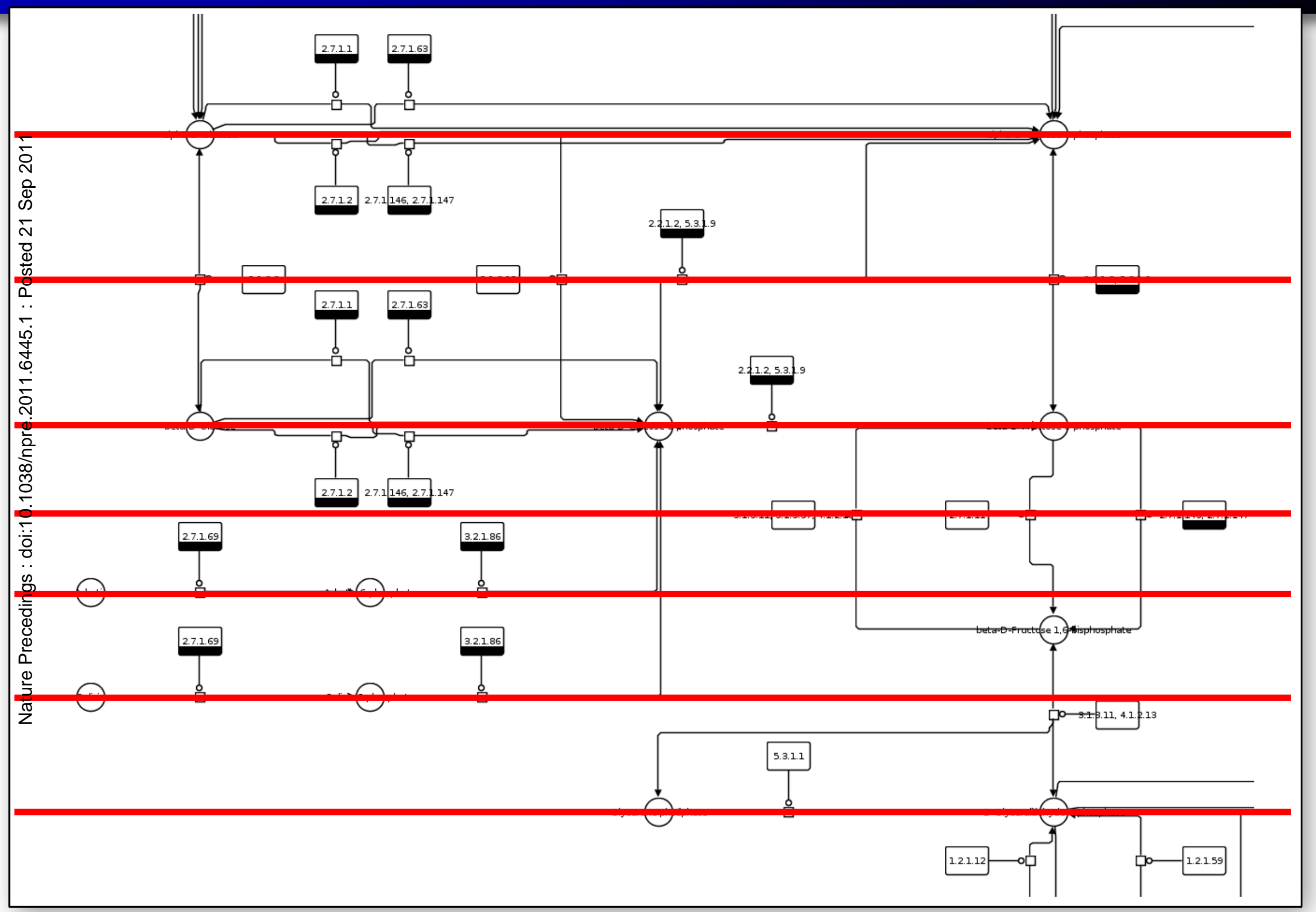




\section{Constrained KEGG Layout}

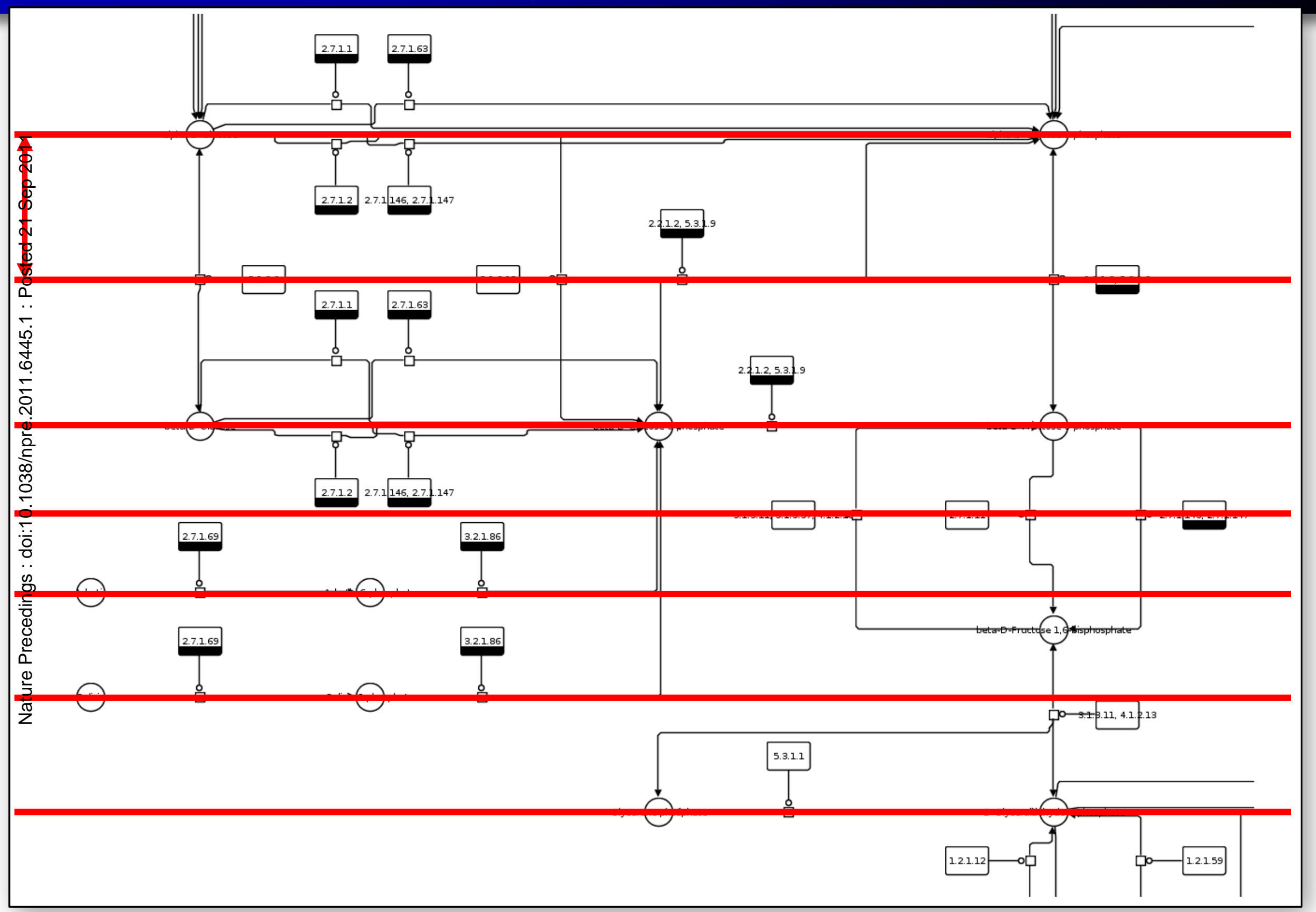




\section{Constrained KEGG Layout}

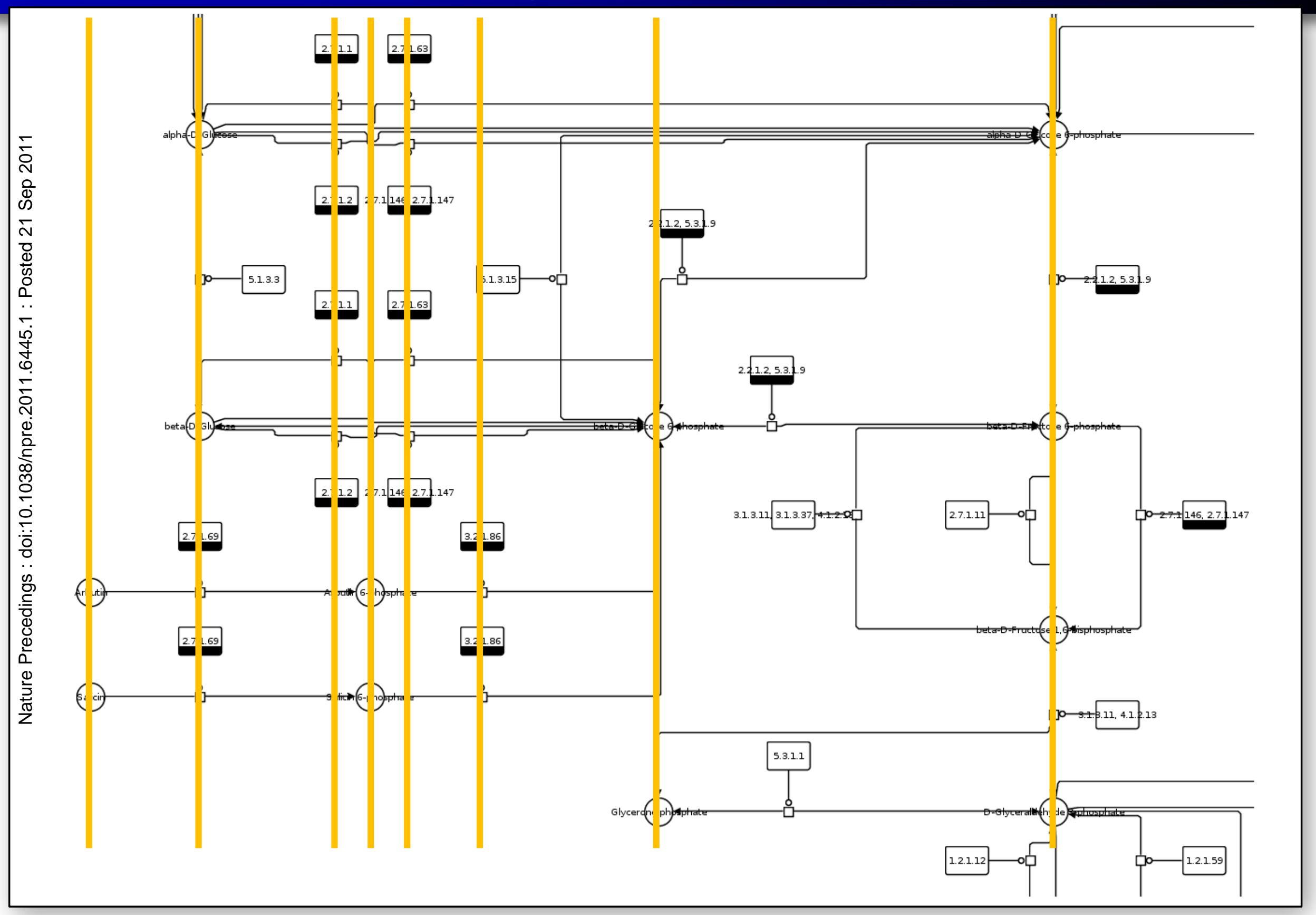




\section{Constrained KEGG Layout}

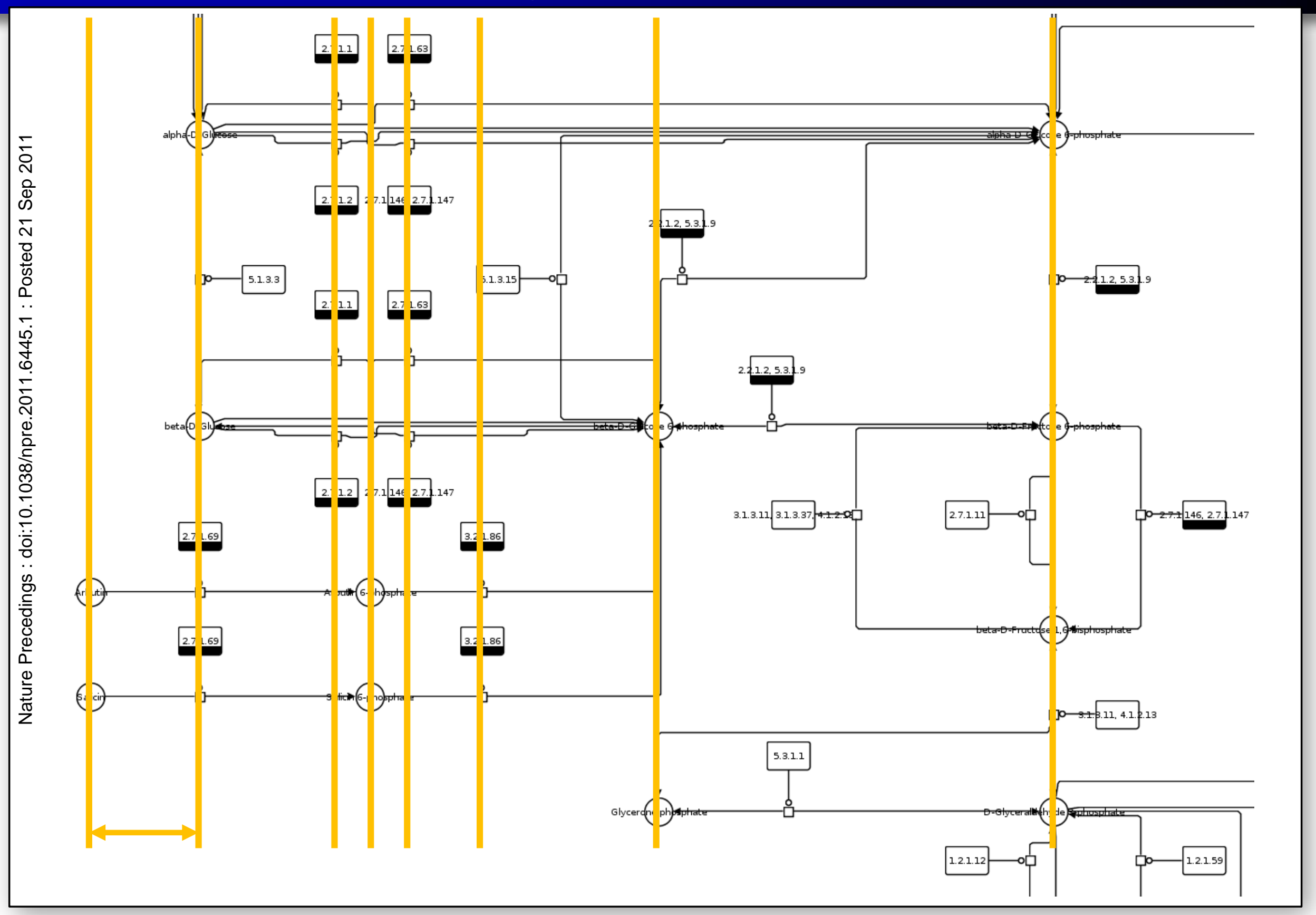




\section{Constrained KEGG Layout}

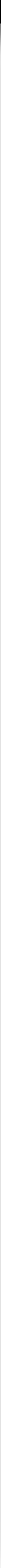




\section{Constrained KEGG Layout}

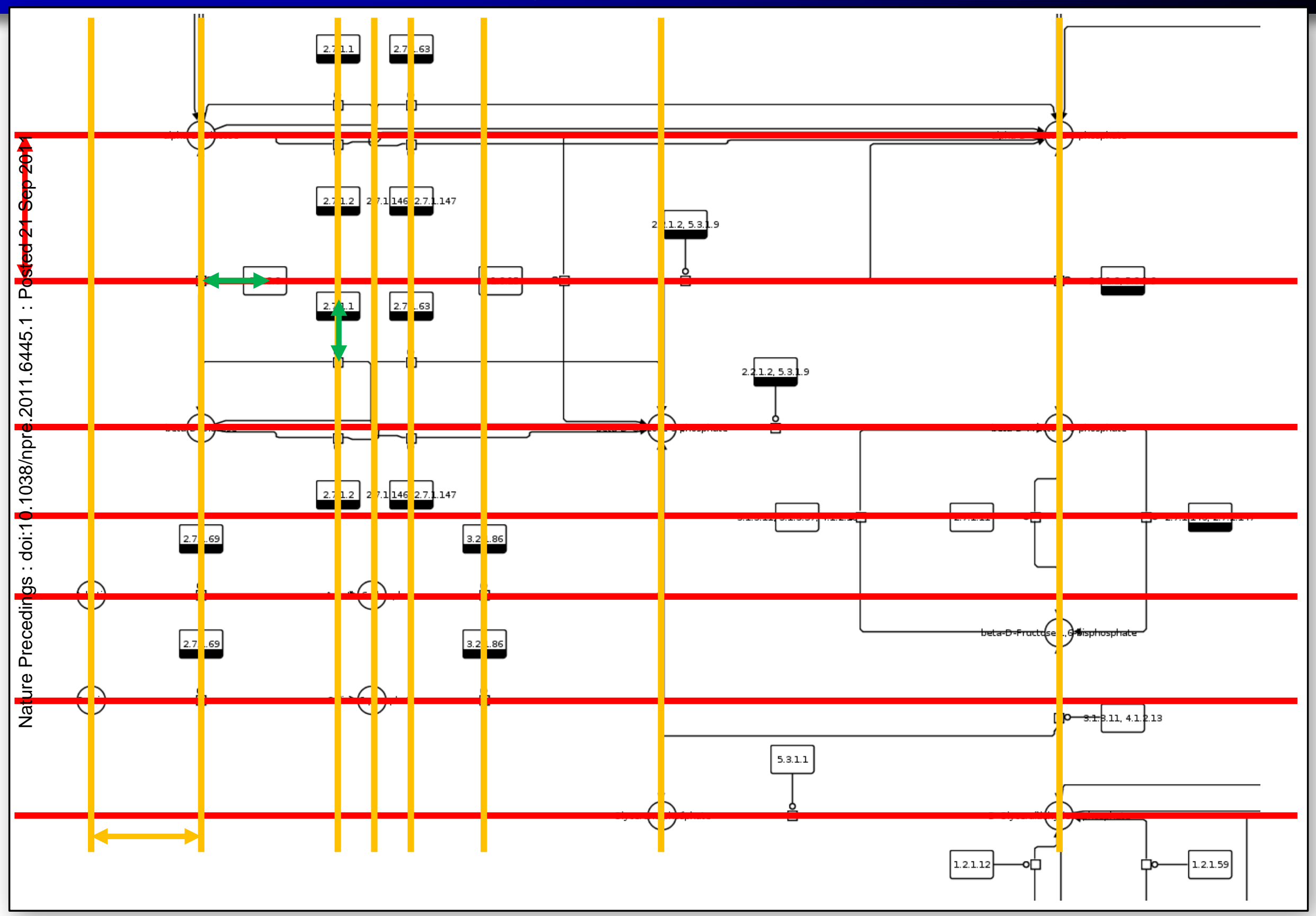




\section{Constrained KEGG Layout}

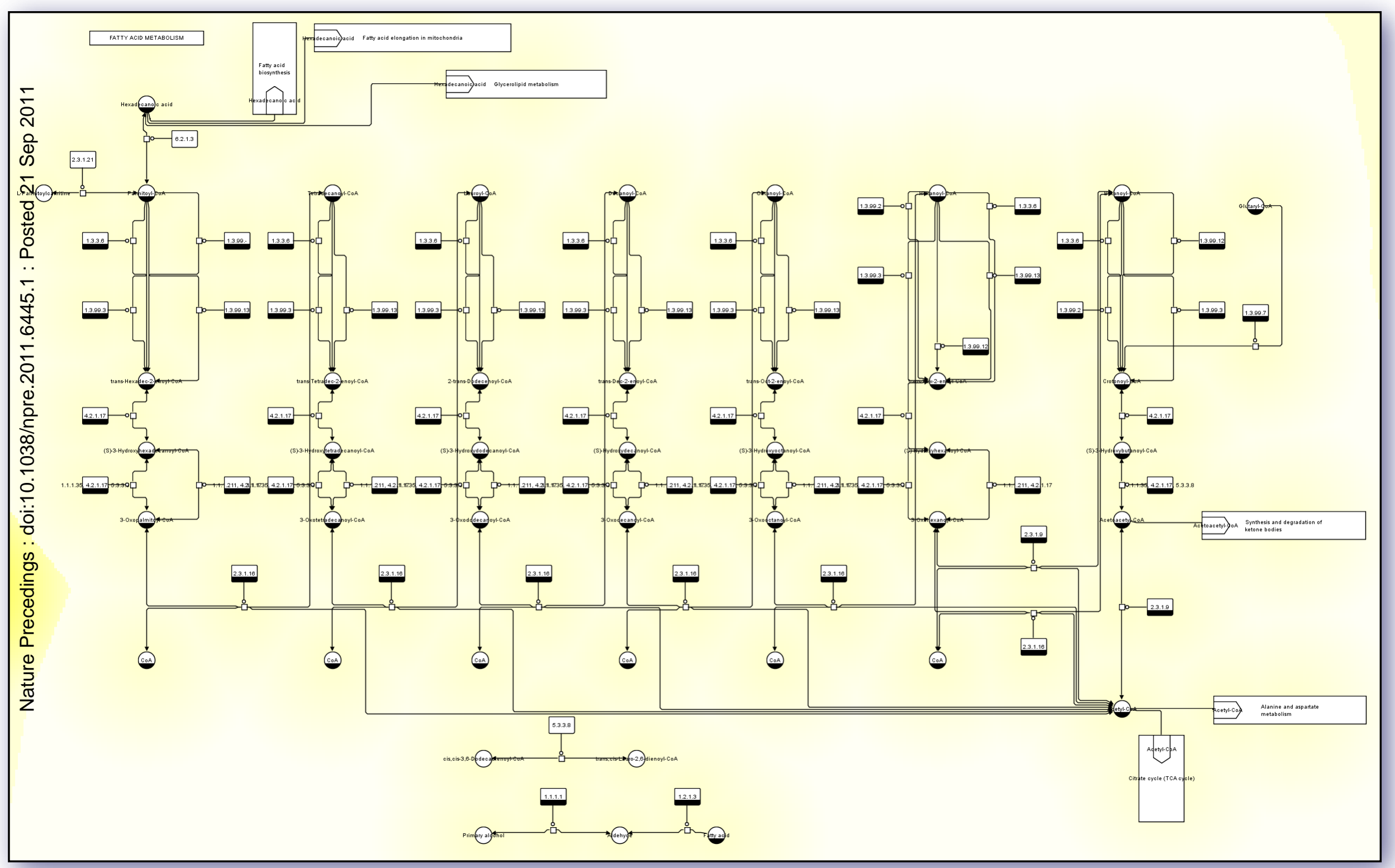




\section{Constrained KEGG Layout}

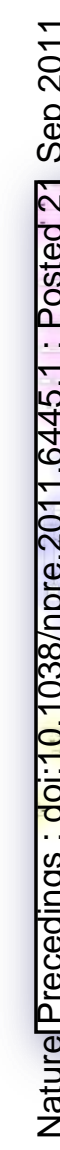




\section{Automatic Layout of SBGN}

- Network layout algorithms = solve given constraints

- Problem: speed

- Solution: Solvers for restricted sets of constraints

- New method based on stress majorisation

- Java library for SBGN

- Joint work with Kim Marriot, Michael Wybrow (both Monash University, Australia) and Tim Dwyer (Microsoft Research) 


\section{Applications: RIMAS}

- Regulatory interaction maps of Arabidopsis seed development

- http://rimas.ipk-gatersleben.de

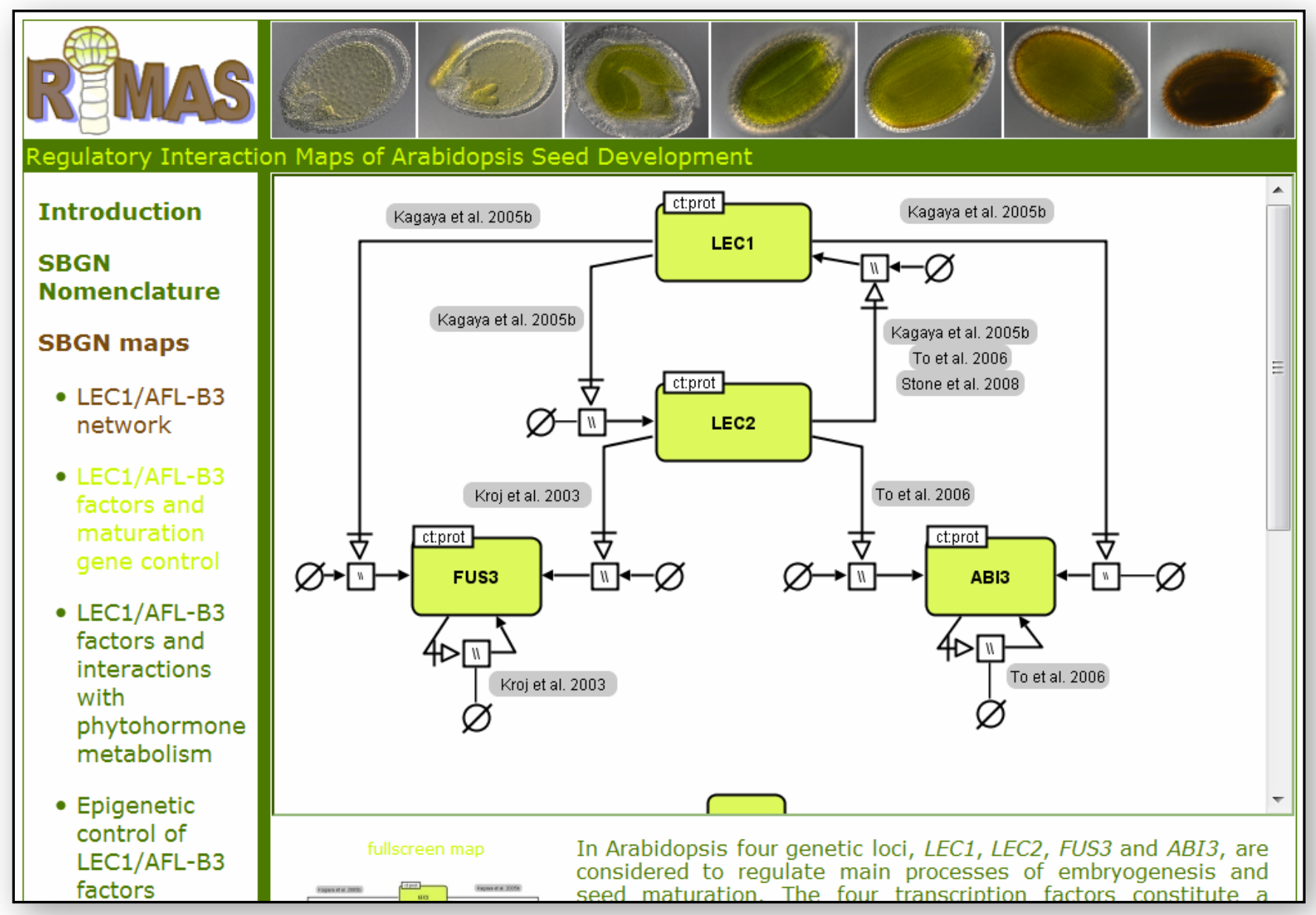




\section{Applications: MetaCrop}

- Information system for diverse information about metabolic pathways in crop plants

- Supports creation of detailed metabolic models

- http://metacrop.ipk-gatersleben.de
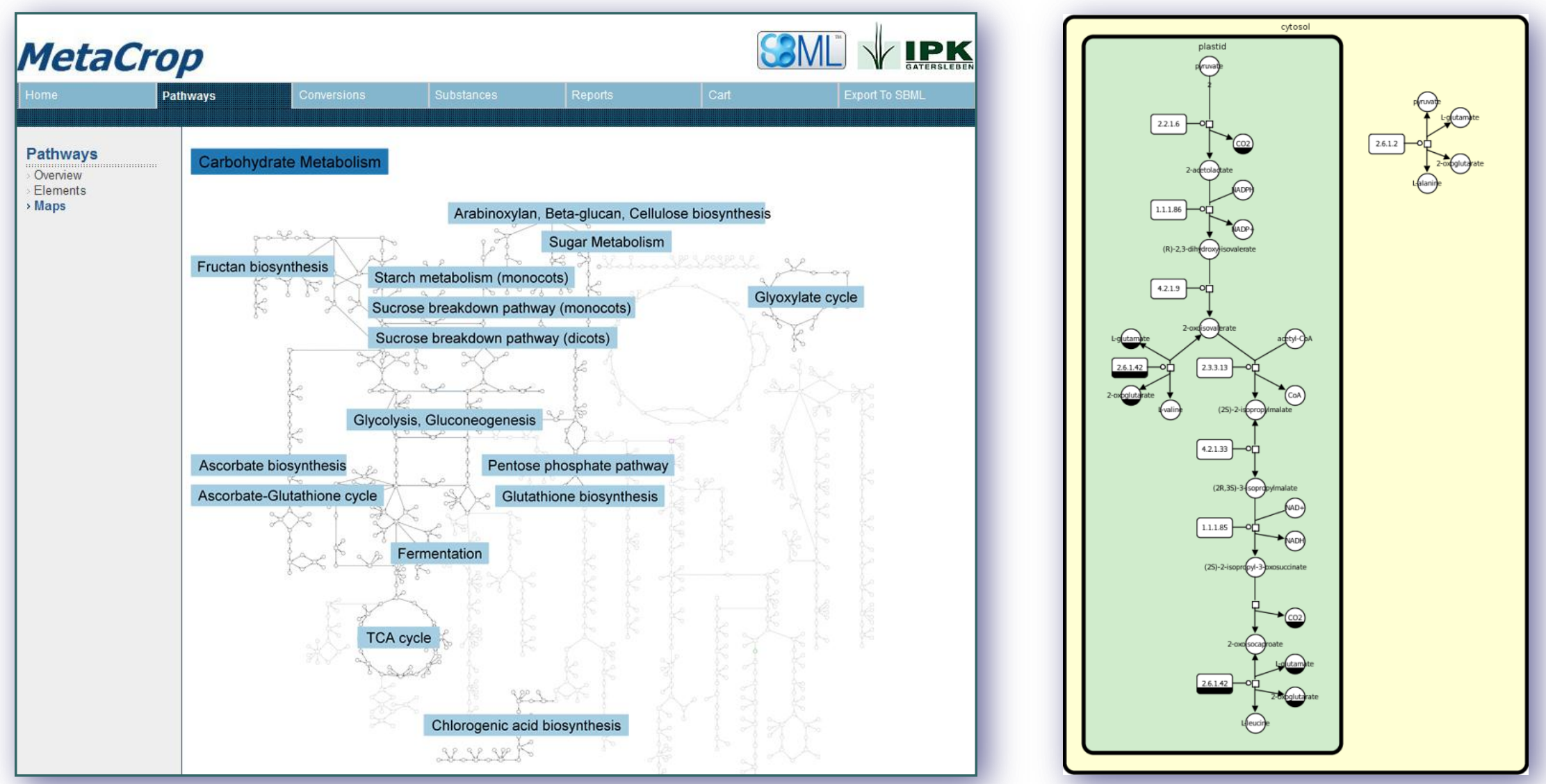


\section{Applications: Maps for Other Groups}
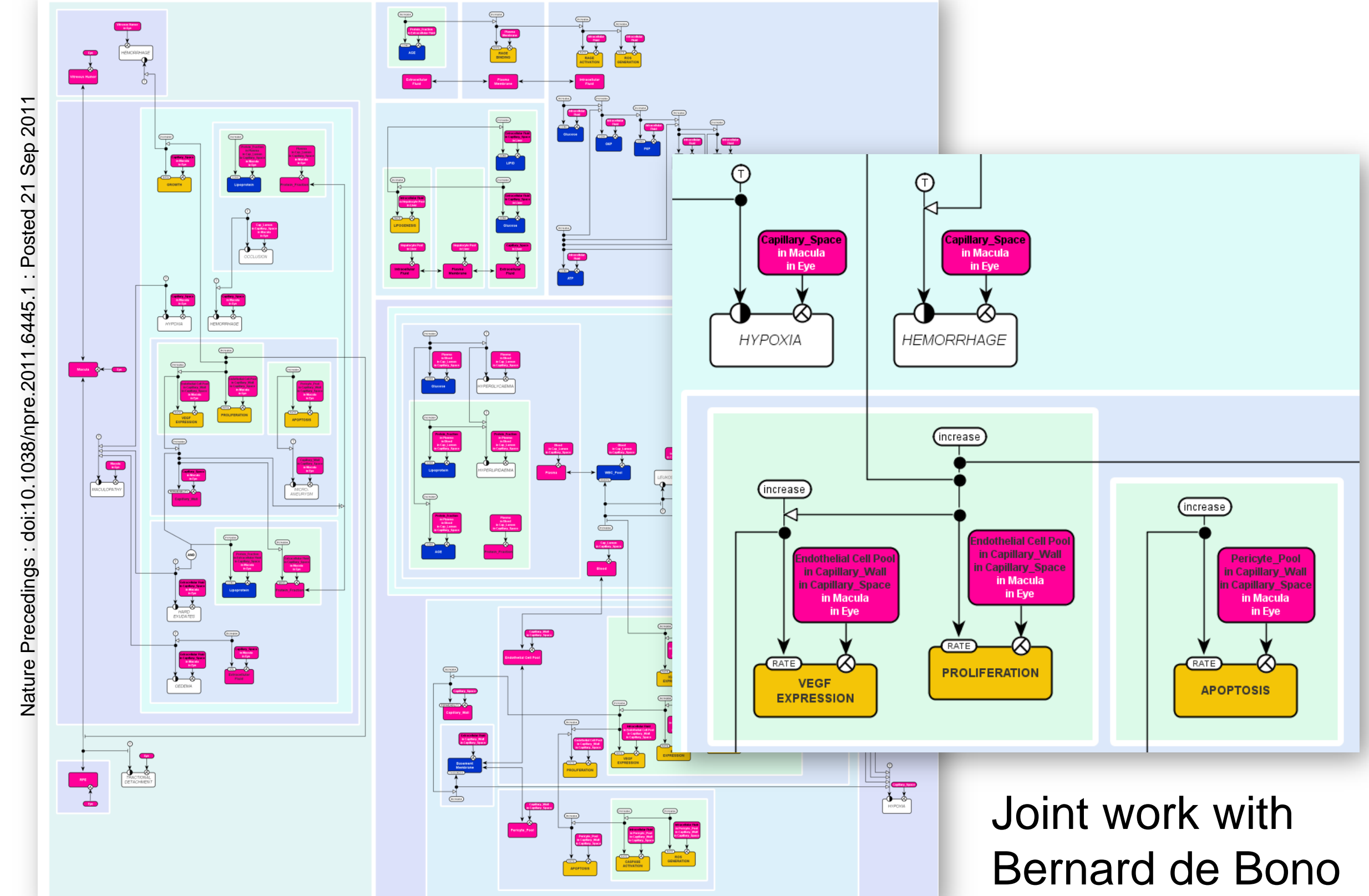


\section{Implementation}

- Add-on to Vanted

- MVC pattern
- Based on extensible, plugin-based graph visualization toolkit Gravisto (group of Franz. J. Brandenburg, Passau University)

- Event management (observer design pattern)

- Java application

- External plug-ins ('Add-ons')

- www.vanted.org

\section{Vanted Plug-ins}

GUI-Components

Input-, Output-

Serializers
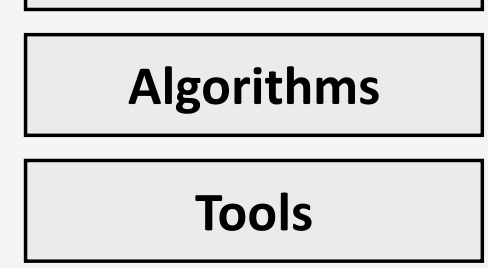
Algorithm Parameters

Editors

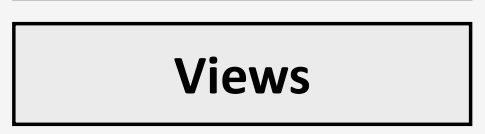

Addon-Manager
Attributes,

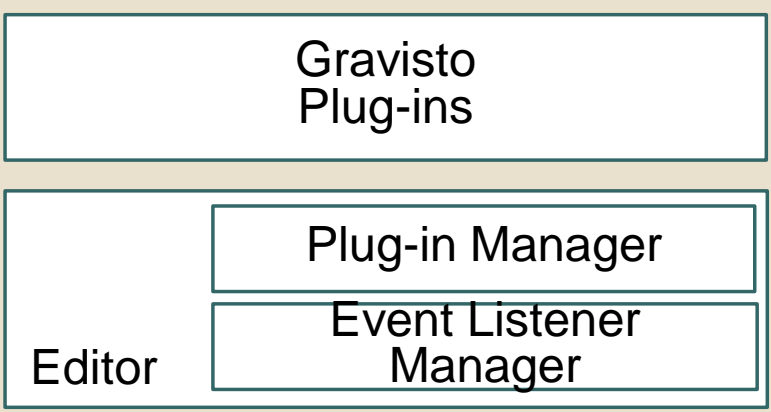

External Libraries
Graph Data Structures

Adj. List

Adj. Matrix

System architecture 


\section{Vanted Add-ons - http://www.vanted.org}

VANTED - VISUALIZATION AND ANALYSIS OF NETWORKS CONTAINING EXPERIMENTAL DATA

VANTED Source Code/ Development Add-ons Literature

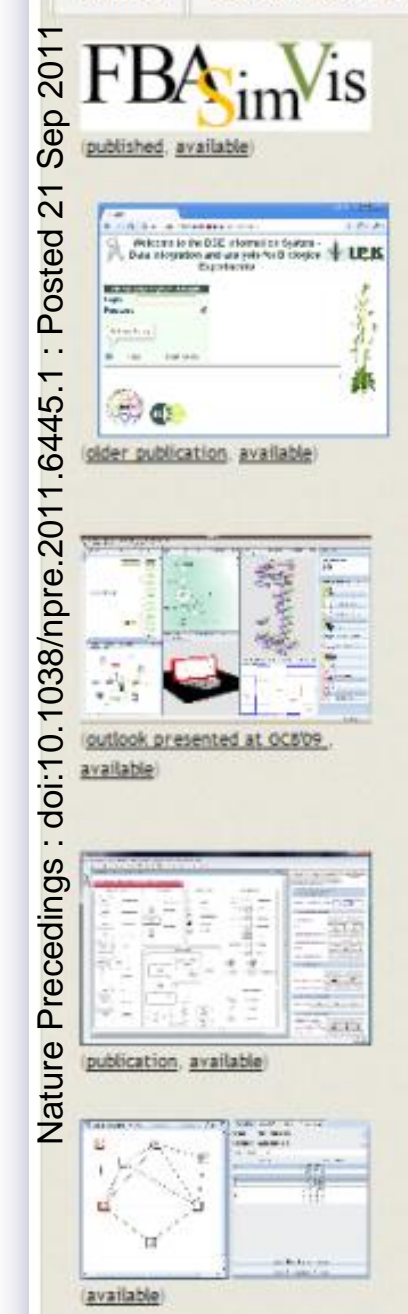

Dynamic visualisation of constraint-based metabolic models

FBA.SimVis is a VANTED Add-on for the constraint-based analysis of metabolic models with special focus on the dynamic and visual exploration of metabolic flux data resulting from model analysis.

DBE2 - Database for Biological Experiments

The database system DBE 2 , is an extension of the original DBE system, which also supported data presentation in the web (example). The improved version of this system, DBEZ may now be used to easily store biclogical experiment data in a central and save place at our institute. It can be accessed directly from within the VANTED tool by instaling a VANTED Add-on (ink). Your experiment data can be easily shared and combined with different datasets using the VANTED and DBE2 systems.

HIVE - Handy Integration and Visualisation of multimodal Experimental Data

In the frame of the SYSSEED project, HIVE combines network-focused Systems Biology approaches with spatio-temporal information. It extends the functionality of VANTED by adding the handling of volumes and images, together with a workspace approach, alowing one to integrate data of different biological data domains. HIVE is written in Java and Java3D and the main tasks are:

- handing of omics data, networks, images and volumes

- integration of different data types using a graph-based workspace

- combining biological data in different ways and

- manifold visualisations of combined data

SBGN-ED - Editing, Translating and Validating of SBGN Maps

SBGN-ED is a VANTED Add-on which alows to create and edit al three types of SBGN maps, that is Process Description, Entity Relationship and Activity Flow, to validate these maps according to the SBGN specifications, to translate maps from the KEGG and MetaCrop pathway databases into SBGN, and to export 5BGN maps into severai file and image formats.

SBGN (Systems Biology Graphical Notation) is an emerging standard for graphical representations of biochemical and celular processes studied in systems biology.

Centilib - Computation of network centratities

Centilib is an Add-on for the computation and investigation of weighted and unweighted centraities in biological networks. Even though Centilib was developed to integrate the computation of centraities into as many tools for the visualisation and analysis of biological networks as possible, it can be used for other types of networks, too.

FluxMap - visual exploration of flux distributions in biological networks

EluxMap is an easy to use tod for the advanced visualization of simulated or measured flux data in biological networks. Flux data import is achieved via a structured template basing on intuitive reaction equations. Flux data is mapped onto any network and visualized using edge thickness. Various visualization options and interaction possibitities enable comparison and visual analysis of 


\section{Many Additional Functions via Vanted}

- Network analysis and visualisation algorithms

- Centralities, motifs, clusters, graph properties

- Layout algorithms, graph editing

- Data mapping (omics data)

- Statistical analysis

- Access to databases, importers, exporters

- Simulation

- 3D visualisation

- And more

- www.vanted.org (Vanted 2.0)

- Open source (GPL) 


\section{Acknowledgements}

- Astrid Junker

- Anja Hartmann

- Eva Grafahrend-Belau

- Hendrik Rohn

- Matthias Klapperstück

- Hendrik Mehlhorn

\section{SBGN-ED}

www.sbgn-ed.org

- Christian Klukas

- Kim Marriott (Monash Univ Melbourne)

- Michael Wybrow (Monash Univ Melbourne)

- Tim Dwyer (Microsoft)

- Franz J. Brandenburg

- libSBGN community

- SBGN community 